\title{
Glucagon Effects on Ischemic Vasodilatation in the Isolated Rat Heart
}

\author{
Mirko Rosic, ${ }^{1,2}$ Suzana Pantovic, ${ }^{1}$ Gvozden Rosic, ${ }^{1}$ Aleksandra Tomic-Lucic, ${ }^{1}$ \\ Tatjana Labudovic, ${ }^{1}$ Vladimir Zivkovic, ${ }^{1}$ and Vladimir Jakovljevic ${ }^{1}$ \\ ${ }^{1}$ Department of Physiology, Medical Faculty, University of Kragujevac, Svetozara Markovica 69, 34000 Kragujevac, Serbia \\ ${ }^{2}$ Research Center of Serbian Academy of Arts and Sciences and the University of Kragujevac, 34000 Kragujevac, Serbia
}

Correspondence should be addressed to Mirko Rosic, mrosic@medf.kg.ac.rs

Received 30 July 2009; Revised 30 October 2009; Accepted 19 January 2010

Academic Editor: Abdel A. Abdel-Rahman

Copyright (c) 2010 Mirko Rosic et al. This is an open access article distributed under the Creative Commons Attribution License, which permits unrestricted use, distribution, and reproduction in any medium, provided the original work is properly cited.

\begin{abstract}
The myocardial reperfusion following ischemia leads to the ischemic vasodilation by affecting the release of various vasoactive substances, such as free radicals, NO, and histamine. In addition, some evidences suggest that glucagon itself may alter the release of those substances. In this study, we investigated the ischemic vasodilation of the isolated rat heart, as well as the concentrations of NO, TBARS, and histamine in the coronary venous effluent either in the presence or in the absence of glucagon. Our results showed that in the presence of glucagon, there was a faster restoration of coronary perfusion pressure during ischemic vasodilation compared to the absence of glucagon $(124 \pm 5.6$ versus $81 \pm 5.2 \mathrm{~s})$ with no apparent changes in TBARS concentration. The glucagon's administration leads to the decreased release of histamine by approximately $35 \%$. Biphasic release of NO in the presence of glucagon initially showed augmentation by $60 \%$, followed by the significant attenuation of $45 \%$.
\end{abstract}

\section{Introduction}

Although the myocardial reperfusion following ischemia can produce the salvage of the ischemic tissue, it may also contribute not only to the endothelial dysfunction and the myocardial cellular injury, but it also may change the release of the biologically active substances, such as the endothelium-derived relaxing factor (NO) [1]. Even though $\mathrm{NO}$ obtained from L-arginine is a free radical per se, it also can quench other free radicals, including the superoxide radicals and it thereby protects both the endothelial cells and the cardiac myocytes $[2,3]$. In addition, NO may stabilize mast cells and inhibit the release of histamine from the both isolated and the resident cardiac mast cells, thus influencing the postischemic myocardial injury [4-6]. Thus, low doses of NO appear to play a key protective role from the myocardial ischemia-reperfusion injury. However, high concentrations of NO exert highly toxic and harmful effect during the ischemia-reperfusion injury [3,7-9]. Beside the fact that NO may stabilize the cardiac mast cells and it consequently decreases the histamine release $[4,5,10]$, earlier results [11] showed a positive feedback relationship between NO and histamine in the regulation of both coronary ischemic vasodilation and the coronary autoregulation.

In our previous work, we evaluated both the dynamic response and the biomechanical properties of the isolated blood vessels in the presence of NO precursor, L-arginine [12]. Our results demonstrated that some relaxation, such as the increase in the diameter of the blood vessel, was evident in the presence of L-arginine. At the same time, the development of the pressure was significantly faster compared to the group without the L-arginine. The analysis of the biomechanical parameters, such as the stress-strain and the shear stress, suggested that the isolated blood vessel became more rigid in the presence of L-arginine.

The myocardial ischemia and the reperfusion have also been suggested to increase the histamine production [13]. The histamine not only exists (to a great extent) within the mast cells and the basophilic leukocytes, but it also could be released by the endothelial cells, the aggregating platelets, lymphocytes and monocytes/macrophages [14,15]. High concentrations of the histamine are present in the cardiac tissue of most animal species, including humans [13] provoking various cellular functions via the stimulation of 
four different G-protein-coupled receptors $\left(\mathrm{H}_{1}, \mathrm{H}_{2}, \mathrm{H}_{3}\right.$, and $\mathrm{H}_{4}$ ). The stimulation of the $\mathrm{H}_{2}$ receptors by the histamine induces both positive inotropic and positive chronotropic effects $[16,17]$. It has been shown that the blockage of the histamine $\mathrm{H}_{2}$ receptors improved the anaerobic metabolism of the myocardium in the ischemic heart disease that has been associated with the reduced myocardial oxygen consumption demonstrating the highly beneficial effect against the ischemia-reperfusion injury. Moreover, the antagonists to the $\mathrm{H}_{2}$-histamine receptors in the mast cells of the heart may reduce the activity of the mast cells, thereby; they may reduce the release of the mast cell's mediators $[13,18,19]$. Even though the blockage of the histamine $\mathrm{H}_{2}$ receptors limited infarct size, the inhibition of the histamine $\mathrm{H}_{1}$ receptors did not demonstrate this beneficial effect [13]. In the heart, the stimulation of the $\mathrm{H}_{1}$ receptors with histamine impairs the atrioventricular (AV) conduction, thus contributing to the development of the ventricular fibrillation in the myocardial ischemia-reperfusion injury [20,21]. Furthermore, the mast cells play a role in the generation of the reactive oxygen species (ROS), such as superoxide anion and hydrogen peroxide, that are responsible for the further increased release of the histamine from the mast cells and vice versa [6]. The histamine effects on the coronary arteries are the result of the multiple actions of this molecule on both smooth muscle cells and the endothelium. Some reports showed different effects of the histamine including both the relaxation and the constriction of the coronary vessels depending on species, dose of the histamine, the diameter of the blood vessels and the initial vessel's tone, as well as the relative location within the coronary circulation [22].

The glucagon is an endogenous polypeptide hormone that exerts both positive inotropic and the chronotropic effects on the myocardial tissue $[23,24]$. The investigations of the glucagon influence on the transport of the radiolabeled histamine in the isolated guinea pig heart demonstrated the elevation of the histamine transport in the presence of glucagon $[25,26]$. Previous findings showed that the cardiac anaphylactic crisis was markedly reduced in the presence of glucagon. Furthermore, these data suggested that the antiarrhythmic action of glucagon during the cardiac anaphylaxis involved the inhibition of histamine release, the vasodilation of the coronary vessels, the increase in the automaticity of the sinoatrial node, and the enhancement of the atrioventricular conduction velocity [27]. Moreover, it has been demonstrated that the glucagon influenced the accumulation of histamine in the vesicles of enterochromaffin-like cells [28]; however, until now there have not yet been reported data explaining the glucagon-induced stimulation of the histamine storage in the heart cells.

We postulated that glucagon can affect the changes of the coronary perfusion pressure during the ischemiareperfusion, by altering the release of $\mathrm{NO}$ and/or histamine in the heart. Therefore, in the present study, we investigated the changes in the coronary perfusion pressure during the ischemia-reperfusion period, as well as the concentrations of nitric oxide, thiobarbituric acid reactive substances (TBARS), and histamine either in the presence or in the absence of glucagon in the coronary venous effluent of the isolated rat heart. Here, we applied the experimental model and originally developed mathematical procedures (12) to describe the dynamic response of the coronary blood vessels.

\section{Material and Methods}

All experiments were performed according to EU (86/609/EEC) and the Local ethical guidelines. Wister rats of both sexes (200-250 g body weight) were killed by the cervical dislocation. The rat hearts were rapidly isolated and retrogradely perused via the aorta, according to the Langendorff's technique at a constant flow $(7-8 \mathrm{~mL} / \mathrm{min} / \mathrm{g}$ wt) of the perfusion buffer containing (in $\mathrm{mM}$ ): $\mathrm{NaCl} 118.1$, $\mathrm{KCl} 4.7, \mathrm{MgSO}_{4} 1.66, \mathrm{NaHCO}_{3} 24.88, \mathrm{KH}_{2} \mathrm{PO}_{4} 1.18$, glucose 5.5 , sodium pyruvate $20, \mathrm{CaCl}_{2} \times 2 \mathrm{H}_{2} \mathrm{O} 2.52$. The perfusate was continuously bubbled with $95 \% \mathrm{O}_{2}$ and $5 \% \mathrm{CO}_{2}$, with the $\mathrm{pH}$ adjusted to 7.4 at $37^{\circ} \mathrm{C}$. The coronary perfusion pressure was measured by a pressure transducer (Elunit, Yugoslavia) and recorded by a Kipp \& Zonen chart recorder (UK). The electrical pacing of the isolated hearts $(3 \mathrm{~V}, 2 \mathrm{~ms}$, $300 \mathrm{bpm}$ ) was performed with the electrodes placed on the right auricles near SA node.

After the equilibration period (20 minutes), the ischemic vasodilation was induced by the interruption of coronary inflow (global ischemia) for 60 seconds. Two to three bouts of the ischemic vasodilation were induced in each heart, both without (the control group, $n=10$ ) and with glucagon solution (the test group, $n=10$ ) approximately 15-20 minutes between the interruptions of the coronary inflow. Glucagon (Sigma, USA) was added in the physiological solution in the final concentration of $400 \mathrm{nM}$, at least 2 minutes before the occlusion, and the addition continued during the reperfusion, until 2 minutes after the reestablishment of the coronary pressure to the preischemic level. The samples of the venous effluent were continuously collected during the reperfusion period and prepared for further analysis: the determination of the histamine concentration, and the levels of NO and TBARS.

The histamine concentration was measured using Shore's fluorometric method [29].

After the coronary occlusion, the coronary pressure decreased near to zero. Following the period of ischemia, the coronary flow was reestablished (the reperfusion period) and the changes in the coronary perfusion pressure (CPP) versus time were recorded.

The experimentally recorded dependence of $\mathrm{CPP}$ on time during the reperfusion period was fitted using an exponential mathematical function:

$$
y=b_{1}\left(1-e^{-b_{2} x}\right),
$$

where $y$ was the CPP (in $\mathrm{mmHg}$ ), $x$ was the time (in s), and $b_{1}$ and $b_{2}$ were the coefficients of this relationship:

(i) $b_{1}$ has units of pressure, and

(ii) $b_{2}$ has units of time ${ }^{-1}$.

This function is shown in the Figure 1. as the CPP versus time curve. The constant $b_{1}$ represents the maximum 


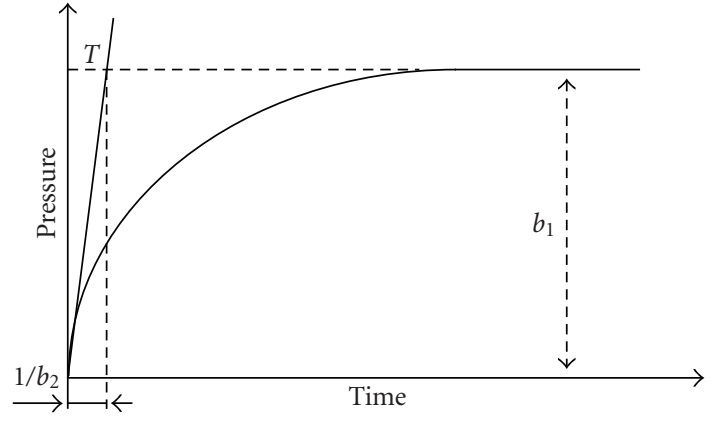

FIGURE 1: Exponential curve $y=b_{1}\left(1-e^{-b_{2} x}\right)$.

developed pressure, that is, the pressure corresponding to the alternate steady state.

We introduced a dominant time constant $(T)$ as the time value corresponding to the cross section point between the asymptote of the exponential curve and the tangent of the exponential curve at the zero point. This constant results from the following function (1):

$$
T=\frac{1}{b_{2}} \text {. }
$$

We considered that the alternate steady state was reached for $t=5 T(t=$ time $)$, because in this case, $e^{-t / T}=e^{-5} \approx 0$, and $y \approx b_{1}$.

Beside the time duration of the CPP restoration (or the flow restoration), the area above the CPP or the flow curve had been commonly used as the parameter for the estimation of CPP or the flow restoration after the ischemic period, by means of the conventional mathematical methods. In this paper, the area above the experimental CPP curve was defined as eAIV (area of ischemic vasodilation).

The area above the fitted curve-fAIV (Figure 2) can be defined by the following function (3):

$$
f \mathrm{AIV}=\int_{0}^{\infty}\left[b_{1}-b_{1}\left(1-e^{-b_{2} x}\right)\right]
$$

and calculated from the following function (4):

$$
f \mathrm{AIV}=\frac{b_{1}}{b_{2}} .
$$

2.1. Nitrite Determination. Nitric oxide was assessed as nitrite and quantified by the spectrophotometric method using the Griess-reagent. $0.5 \mathrm{~mL}$ of the perfusate was precipitated with $200 \mu \mathrm{L}$ of $30 \%$ sulfosalicylic acid, vortexed for 30 minutes and centrifuged at $3000 \times \mathrm{g}$. The equal volumes of the supernatant and Griess's reagent, containing $1 \%$ sulfanilamide in $5 \%$ phosphoric acid $/ 0.1 \%$ naphthalene ethylenediamine-dihydrochloride were added and incubated for 10 minutes in the dark and read at $543 \mathrm{nmol} / \mathrm{l}$. The nitrite levels were calculated using sodium nitrite as the standard [30].

2.2. Index of Lipid Peroxidation (Thiobarbituric Acid Reactive Substances-TBARS). The degree of lipid peroxidation in the
TABLE 1: Histamine concentrations in the control (without glucagon) and test (in the presence of glucagon) groups taken during preischemic and reperfusion periods.

\begin{tabular}{lcc}
\hline & Control group & Test group \\
\hline $\begin{array}{l}\text { Histamine } \\
\text { concentration in } \\
\text { preischemic period } \\
(\mu \mathrm{M})\end{array}$ & $4.6 \pm 0.8 \cdot 10^{-8}$ & $5.1 \pm 0.6 \cdot 10^{-8}$ \\
$\begin{array}{l}\text { Histamine } \\
\text { concentration within } \\
\text { reperfusion period } \\
(\mu \mathrm{M})\end{array}$ & $10.4 \pm 0.9 \cdot 10^{-8 * \dagger}$ & $6.8 \pm 0.5 \cdot 10^{-8 * \dagger}$ \\
\hline
\end{tabular}

Values are represented as mean \pm S.E.M.

(*) Represents significantly different values between control and test groups, $P<.01 ; n=10$.

$\left({ }^{\dagger}\right)$ Represents significantly different values between histamine concentrations in preischemic and reperfusion periods, $P<.01 ; n=10$.

coronary venous effluent was estimated by the measurement of thiobarbituric acid reactive substances (TBARS) using 1\% TBA (thiobarbituric acid) in $0.05 \mathrm{NaOH}$ incubated with the coronary effluent at $100^{\circ} \mathrm{C}$ for 15 minutes and read at $530 \mathrm{~nm}$. Krebs-Henseleit solution was used as a blank probe [31].

2.3. Statistical Analysis. Data are presented as mean \pm S.E.M. (standard error of the mean). Statistical analysis was performed by using the multifactorial analysis of the variance for repeated measurements between the subject factors as well as Bonferroni-test. $P$ values less than .05 were considered to be significant. Also, the data were analyzed using Student's $t$-test, where $P$ value of $<.05$ was considered as statistically significant.

\section{Results}

To investigate the effect of the glucagon on the concentration of the histamine in the coronary venous effluents, the levels of histamine were measured both in the control and in the test groups either in the absence $(n=10)$ or in the presence $(n=10)$ of the glucagon, respectively. Our results demonstrated no statistically significant differences in the levels of the histamine concentration both in the control and the test groups, as shown in the Table 1. On the other hand, there was a significant increase of the histamine release both in the control (126\%) and the test groups (33\%) during the reperfusion period compared to the preischemic levels. The increase of the histamine concentrations achieved the maximal values within 30 to $90 \mathrm{~s}$ in the control group and within 30 to $60 \mathrm{~s}$ in the test group during the reperfusion period. In addition, our results indicated a significant decrease of the histamine release in the presence of glucagon during the reperfusion period compared to the control group.

Furthermore, we measured the levels of $\mathrm{NO}$ in the coronary venous effluent, collected both during preischemic and the reperfusion periods, both in the control $(n=10)$ and the test $(n=10)$ groups, as presented in the Figure 3 . The 


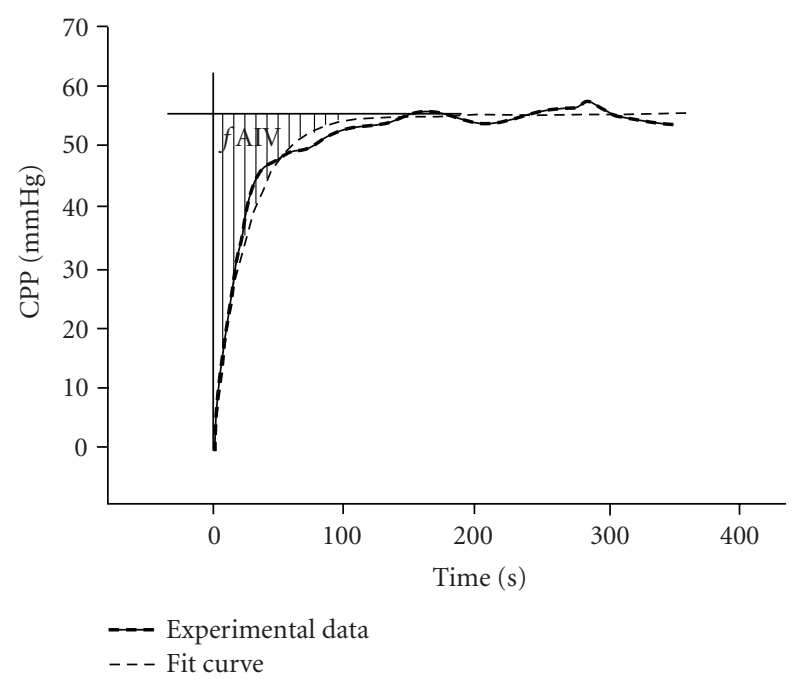

(a)

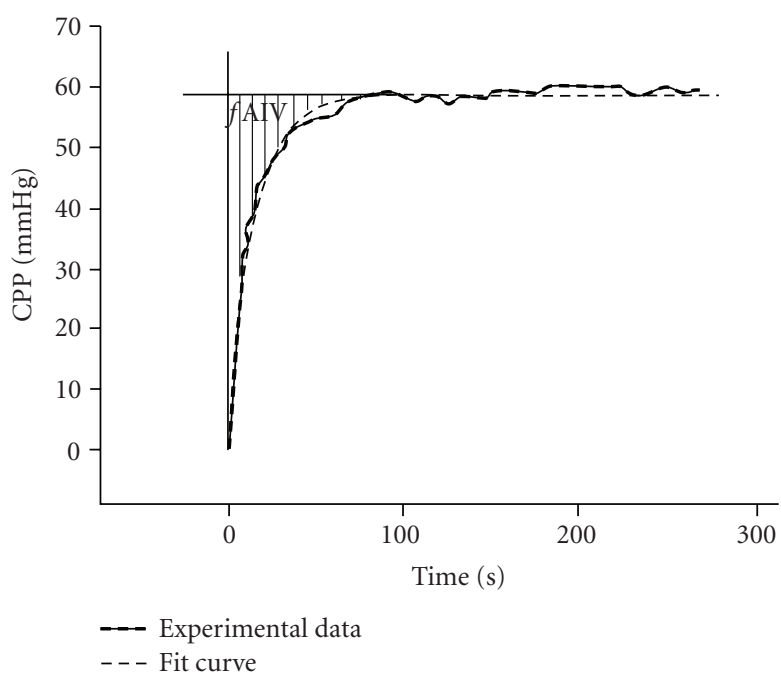

(b)

FIGURE 2: Restoration of CPP (original trace) in the absence (a) and in the presence (b) of glucagon ( $f$ AIV is area of ischemic vasodilation above the fitted CPP curve).

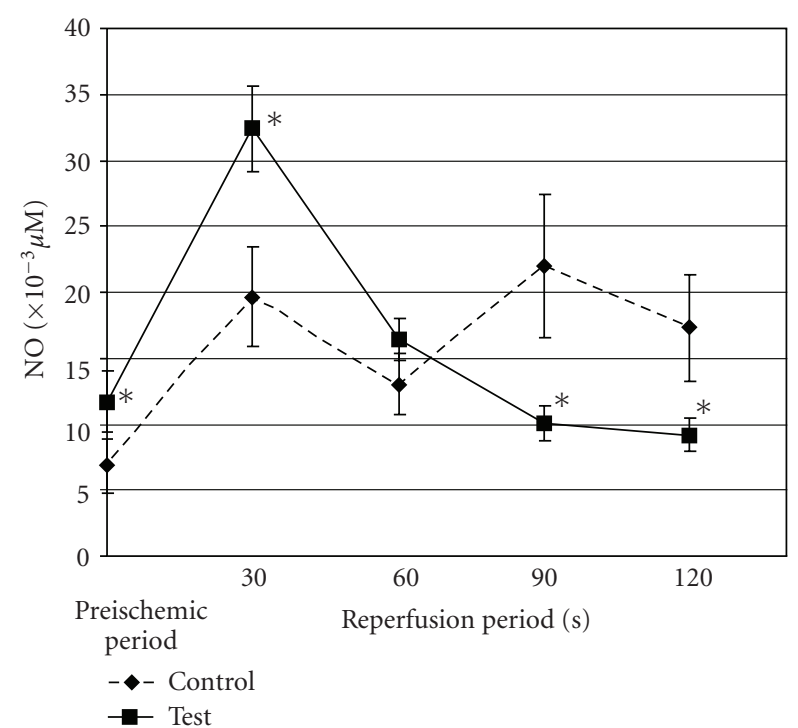

FIGURE 3: NO concentrations in the coronary venous effluent collected during preischemic and reperfusion period in the control and test groups. $*$ represents significantly different values between corresponding values in control and test groups, $P<.01 ; n=10$.

results showed the increase of $\mathrm{NO}$ concentrations in both groups during the reperfusion period. Also, the levels of NO in the test group were significantly elevated $(71 \%)$ during the preischemic period in comparison to the control group.

The elevated concentrations of NO in the control group collected during the reperfusion period showed two peak values:

(i) the first peak was evident 30 seconds following the reperfusion period with the increase of $187 \%$, and (ii) the second peak showed 90 seconds following the reperfusion period with the increase of $221 \%$.

On the other hand, the NO level in the test group achieved one peak value within first 30 seconds of the reperfusion period. After reaching the peak value, the NO level in the test group gradually decreased until the end of the reperfusion period.

Moreover, within first 30 seconds of the reperfusion period, the NO levels in the test group were initially significantly higher $(60 \%)$ but eventually reached significantly lower levels (45\%) within first 90 seconds of the reperfusion period, compared to the corresponding $\mathrm{NO}$ levels in the control group.

Next, we investigated the concentrations of TBARS in the samples collected during both the preischemic and the reperfusion periods, both in the control and in the test groups. Our results showed that the concentrations of TBARS in the samples collected during the reperfusion period were greatly elevated in comparison to the preischemic levels, both in the control group $(202.6 \%, n=10)$ and in the test group $(226.7 \%, n=10)$, as shown in the Table 2 . The highest TBARS concentrations were achieved within the first 30 seconds of the reperfusion period in both groups. In addition, our results showed no statistically significant differences in the TBARS levels neither in the control nor in the test group both during the preischemic and the reperfusion periods.

Further, we analyzed the experimental CPP-time curves using a method as described in Materials and Methods. The values of $5 T$, the $b_{1}$ and $b_{2}$ coefficients of the fitted experimental data (coefficient of correlation was $0.998 \pm$ $0.002)$ both in the control $(n=10)$ and in the test $(n=$ 1) groups were shown in the Table 3 . Our analyzed data indicated significant increase of the coefficient $b_{2}$ in the test group compared to the control group. However, the $5 T$ value 
TABLE 2: TBARS levels in control (without glucagon) and test (in the presence of glucagon) groups taken during preischemic and reperfusion periods.

\begin{tabular}{lcc}
\hline & Control group & Test group \\
\hline $\begin{array}{l}\text { TBARS concentration } \\
\text { in preischemic period } \\
(\mathrm{mM})\end{array}$ & $6.49 \pm 3.44 \cdot 10^{-6}$ & $5.61 \pm 3.41 \cdot 10^{-6}$ \\
$\begin{array}{l}\text { TBARS concentration } \\
\text { within reperfusion } \\
\text { period }(\mathrm{mM})\end{array}$ & $19.64 \pm 3.17 \cdot 10^{-6}$ & $18.33 \pm 2.15 \cdot 10^{-6}$ \\
\hline
\end{tabular}

Values are represented as mean \pm S.E.M $(n=10)$.

TABle 3: Calculated mathematical parameters $\left(b_{1}\right.$ and $\left.b_{2}\right)$ in the control and test groups, and time within which the maximal pressure is developed (taken as $5 T$ ).

\begin{tabular}{lcc}
\hline Parameter & Control group & Test group \\
\hline$b_{1}$ & $54.94 \pm 4.2$ & $58.28 \pm 3.7$ \\
$b_{2}$ & $0.04 \pm 0.003$ & $0.062 \pm 0.004^{*}$ \\
$5 T(\mathrm{~s})$ & $125.4 \pm 5.6$ & $81.7 \pm 5.2^{*}$ \\
\hline
\end{tabular}

Values are represented as mean \pm S.E.M.

$\left.{ }^{*}\right)$ represents significantly different values between control and test group, $P<.01 ; n=10$.

in the test group was significantly lower in comparison to the value of the control group. Moreover, our results showed no great difference in $b_{1}$ coefficients between the control and the test groups of the animals.

When analyzing the $f$ AIV in both the control and the test groups, our results showed that $f$ AIV in the control group was $1373 \pm 33$, while in the presence of glucagon this area was significantly lower $(940 \pm 28)$. At the same time the values of $e$ AIV both in the control group $(1360 \pm 29)$ and the test group $(951 \pm 30)$ were not significantly different in comparison to the corresponding $f$ AIV values.

\section{Discussion}

The restoration of the coronary perfusion pressure during the reperfusion period in our experiments had an exponential form rather than the step function, indicating that the ischemic vasodilation occurred within this period.

In this study, we investigated the ischemic vasodilation of the isolated rat heart, as well as the NO, TBARS, and histamine concentrations in the coronary venous effluent either in the presence or in the absence of glucagon. We used a new approach [12] in the ex vivo experiments considering not only the end points of the vessels response, but also the dynamics of this response. In order to elucidate the mentioned phenomena, we described the dynamic behavior of the coronary blood vessel during the ischemic vasodilation by proposing a mathematical relation where the parameters were evaluated through the relationships of the coronary pressure-time curves with the experimental data. The coronary pressure-time curves were fitted using the simple exponential mathematical function in the way that both $b_{1}$ and $b_{2}$ coefficients had the physical meaning. In this function, the first coefficient $-b_{1}$-numerically described the maximal developed coronary pressure that corresponded to the pressure in the alternate steady state. The second coefficient $-b_{2}$ - was relevant for the description of the vessel transition response between the alternate steady states. In our previous work [12], we demonstrated that these coefficients were highly sensitive parameters to the conditions of the blood vessel dynamics.

The mathematical analysis of the CPP dynamics showed no significant differences in the $b_{1}$ coefficients (Table 3 ) both in the control and in the test groups of animals. This result revealed that the maximal coronary perfusion pressure during the ischemic vasodilation was similar in both groups of animals. On the contrary, the $b_{2}$ coefficient in the test group was significantly higher compared to the $b_{2}$ coefficient of the control group, indicating the alteration in the dynamic response during the ischemic vasodilation in the presence of the glucagon. In addition, the time interval for the development of the maximal CPP during the alternate steady state was 125 seconds in the control group and it was significantly higher compared to the maximal CPP developmental period of 82 seconds in the test group. Therefore, these results demonstrated a faster increase of the coronary pressure in the glucagon-treated hearts (Table 3).

The values of $f$ AIV in the presence of the glucagon were significantly lower compared to the $f$ AIV values of the control group confirming the glucagon alteration of the ischemic vasodilation in our experimental design. On the other hand, the fAIV values were not notably different from the eAIV values in both groups, demonstrating the logical consequence of the extremely high coefficient of the correlation $\left(R^{2}=0.998 \pm 0.002\right)$ between the fitted and the experimental CPP curves (Figure 2).

In addition, we calculated the Index of lipid peroxidation by measuring the levels of thiobarbituric acid reactive substances-TBARS (the known markers of the oxidative stress). Our data demonstrated significantly the elevated levels of TBARS within 2 minutes of the reperfusion both in the control and the test groups compared to the preischemic levels (Table 2). These findings were in accordance with the previous reports $[1,8,32]$. Also, our data demonstrated no apparent differences in the TBARS levels between the control and the test groups, during both the preischemic and the ischemia-reperfusion periods. These results indicated that the glucagon administration did not affect the TBARS levels in the isolated rat heart during both the preischemic and the ischemia-reperfusion periods in our experimental conditions.

Investigation of the NO level both before and during the ischemic vasodilation in the presence and in the absence of the glucagon clearly demonstrated that:

(1) the NO levels during the preischemic period were significantly elevated in the presence of glucagon compared to the control group;

(2) the NO level within the first 30 seconds of the reperfusion period was significantly higher, and after 90 seconds it was significantly lower in the test group, compared to the corresponding NO levels in the control group. 
These findings were similar to the previous data reporting that the nitrite levels were higher under the glucagon treatment in the rat hepatocyte culture [33] and that the NO levels increased during the ischemia-reperfusion conditions, as well $[7,9,34,35]$. Our experimental data also showed no apparent changes in the preischemic CPP values measured continuously during the glucagon administration. These results indicated that the elevated preischemic NO level in the test group did not significantly affect the coronary vessel diameters in our experimental conditions. Even though the elevated preischemic NO level did not have the significant effects on the histamine release, its role in influencing the mast cells stabilization [6] and consequently reducing the histamine release in the test group during ischemic vasodilation should not be neglected.

Our previous findings [12] demonstrated the influence of L-arginine on the faster development of the perfusion pressure in the isolated blood vessels. Although L-arginine in the previous investigation relaxed the smooth muscle in the blood vessel wall, the introduction of the hydrostatic pressure caused the blood vessel wall to become more rigid. These findings indicated that the increase of the NO release might not only induce the vasodilation, but it also might affect the biomechanical properties of the blood vessel wall. Even though the vasodilation occurred at the alternate steady states, the transient or the dynamic response of the blood vessel showed faster development of the pressure.

It is well known that the histamine release is elevated during the ischemic vasodilation and its concentration is increased during the ischemia [36]. Our results in this study confirmed these findings. Also, our results showed that the histamine liberation during the ischemic vasodilation was significantly reduced in the presence of glucagon in the isolated rat heart. These data were in the accordance with the previous reports describing the influence of the glucagon on the inhibition of the histamine release during the anaphylactic response in a guinea-pig isolated heart and the protective effects of the glucagon pretreatment during cardiac anaphylaxis [27].

Changes in both $\mathrm{NO}$ and histamine releases during both the ischemic vasodilation and the coronary autoregulation suggested a positive feedback between NO and histamine [11]. This may explain the biphasic NO release in our control group. The initial increase of $\mathrm{NO}$ within the first 30 seconds of the reperfusion period may lead to the increase of histamine release reaching the peak values between 30 90 seconds. Furthermore, the histamine stimulation of the endothelial histamine receptors produced the second peak of the NO release, by positive feedback mechanism, after 90 seconds of the reperfusion.

As mentioned earlier, the NO level during the preischemic period was significantly elevated in the presence of glucagon. At the same time, there was neither a change in the CPP nor in the basal (preischemic) histamine concentrations. Even though the preischemic NO level was significantly elevated, the amount of NO released seemed to be insufficient to dilate the coronary blood vessels, since there was no change in the CPP at that time. On the other hand, the increased amount of NO might be sufficient to stabilize mast cells, therefore influencing the lack of the positive feedback relationship between $\mathrm{NO}$ and the histamine in the presence of glucagon. This could be the cause of the significantly lower NO release in the test group 90 seconds following the ischemic vasodilation.

\section{Conclusions}

In the summary, the influence of the glucagon on the dynamic responses of the coronary vessels of the isolated rat heart was uncertain during the ischemic vasodilation. In this study, our results showed a faster restoration of the coronary perfusion pressure during the reperfusion period in the presence of glucagon compared to the control group. Therefore, one of the possible postulated mechanism(s) explaining this process could be as following:

(i) the glucagon increases NO release both during the preischemic period and within the first 30 seconds of the ischemic vasodilation;

(ii) NO stabilizes the mast cells and prevents the histamine release during the ischemic vasodilation;

(iii) further, the decreased histamine release then leads to the attenuated stimulation of the endothelial histamine receptor $\left(\mathrm{H}_{1}\right)$ and consequent decrease of the NO release, thus switching off this vasodilatory mechanism;

(iv) simultaneously, the decreased histamine release leads to the attenuation of the $\mathrm{H}_{2} / \mathrm{H}_{3}$ mediated coronary vasodilation in the presence of glucagon;

(v) as a result, the duration of the ischemic vasodilation is significantly shorter in the presence of glucagons;

(vi) moreover, the increased NO release within the first 30 seconds of the ischemic vasodilation leads to the vasodilation in the presence of glucagon that in turn may also increase the rigidity of the coronary blood vessels, generating the faster development of CPP.

Finally, even though the mechanism(s) of the glucagon influences on the coronary vessels during the ischemic vasodilation is still unclear, our results clearly indicate the key involvement of both histamine and NO during this process.

\section{References}

[1] P. S. Tsao, N. Aoki, D. J. Lefer, G. Johnson III, and A. Lefer, "Time course of endothelial dysfunction and myocardial injury during myocardial ischemia and reperfusion in the cat," Circulation, vol. 82, no. 4, pp. 1402-1412, 1990.

[2] G. M. Rubanyi, E. H. Ho, E. H. Cantor, W. C. Lumma, and L. H. Parker-Botelho, "Cytoprotective function of nitric oxide: inactivation of superoxide radicals produced by human leukocytes," Biochemical and Biophysical Research Communications, vol. 18, no. 2, pp. 1392-1397, 1991.

[3] X. S. Li, Y. Uriuda, Q. D. Wang, R. Nordlander, P. O. Sjoquist, and J. Pernow, "Role of L-arginine in preventing myocardial and endothelial injury following ischaemia/reperfusion in the rat isolated heart," Acta Physiologica Scandinavica, vol. 156, no. 1, pp. 37-44, 1996. 
[4] E. Masini, S. Bianchi, L. Mugnai, et al., "The effect of nitric oxide generators on ischemia reperfusion injury and histamine release in isolated perfused guinea-pig heart," Agents and Actions, vol. 33, no. 1-2, pp. 53-56, 1991.

[5] E. Masini, M. G. Di Bello, A. Pistelli, et al., "Generation of nitric oxide from nitrovasodilators modulates the release of histamine from mast cells," Journal of Physiology and Pharmacology, vol. 45, no. 1, pp. 41-53, 1994.

[6] M. Singh and H. K. Saini, "Resident cardiac mast cells and ischemia-reperfusion injury," Journal of Cardiovascular Pharmacology and Therapeutics, vol. 8, no. 2, pp. 135-148, 2003.

[7] A. V. Gourine, A. T. Gonon, and J. Pernow, "Involvement of nitric oxide in cardioprotective effect of endothelin receptor antagonist during ischemia-reperfusion," American Journal of Physiology, vol. 280, no. 3, pp. H1105-H1112, 2001.

[8] S. Bertuglia and A. Giusti, "Microvascular oxygenation, oxidative stress, NO suppression and superoxide dismutase during postischemic reperfusion," American Journal of Physiology, vol. 285, no. 3, pp. H1064-H1071, 2003.

[9] B. I. Jugdutt, "Nitric oxide and cardioprotection during ischemia-reperfusion," Heart Failure Reviews, vol. 7, no. 4, pp. 391-405, 2002.

[10] P. Vinay and M. Singh, "Possible role of nitric oxide and mast cells in endotoxin-induced cardioprotection," Pharmacological Research, vol. 43, no. 1, pp. 39-45, 2001.

[11] M. Kostic and M. Petronijevic, "Interplay of nitric oxide and histamine in the regulation of coronary reactive hyperemia and coronary autoregulation," Agents and Actions, vol. 45, no. 1, supplement, pp. 145-149, 1995.

[12] M. Rosic, S. Pantovic, V. Rankovic, Z. Obradovic, N. Filipovic, and M. Kojic, "Evaluation of dynamic response and biomechanical properties of isolated blood vessels," Journal of biochemical and Biophysical Methods, vol. 70, no. 6, pp. 966972, 2008.

[13] H. Asanuma, T. Minamino, A. Ogai, et al., "Blokade of histamine $\mathrm{H}_{2}$ receptors protects the heart against ischemia and reperfusion injury in dogs," Journal of Molecular and Cellular Cardiology, vol. 40, no. 5, pp. 666-674, 2006.

[14] A. A. Wolff and R. Levi, "Histamine and cardiac arrhythmias," Circulation Research, vol. 58, no. 1, pp. 1-16, 1986.

[15] M. A. Beaven, "Our perception of the mast cell from Paul Ehrlich to now," European Journal of Immunology, vol. 39, no. 1, pp. 11-25, 2009.

[16] G. R. Hageman, F. Urthaler, J. H. Isobe, and T. N. James, "Chronotropic and dromotropic effects of histamine on the canine heart," Chest, vol. 75, no. 5, pp. 597-604, 1979.

[17] L. B. Hough, "Genomics meets histamine receptors: new subtypes, new receptors," Molecular Pharmacology, vol. 59, no. 3, pp. 415-419, 2001.

[18] J. Kim, A. Ogai, S. Nakatani, et al., "Impact of blockade of histamine $\mathrm{H}_{2}$ receptors on chronic heart failure revealed by retrospective and prospective randomized studies," Journal of the American College of Cardiology, vol. 48, no. 7, pp. 1378$1384,2006$.

[19] G. Francis and W. Tang, "Histamine, mast cells and heart failure," Journal of the American College of Cardiology, vol. 48, no. 7, pp. 1385-1386, 2006.

[20] R. Levi and J. O. Kuye, "Pharmacological characterization of cardiac histamine receptors: sensitivity to $\mathrm{H}_{1}$ receptor antagonists," European Journal of Pharmacology, vol. 27, no. 3, pp. 330-338, 1974.

[21] E. Hatta, K. Yasuda, and R. Levi, "Activation of histamine $\mathrm{H}_{3}$ receptors inhibits carrier-mediated norepinephrine release in a human mode of protracted myocardial ischemia," Journal of Pharmacology and Experimental Therapeutics, vol. 283, no. 2, pp. 494-500, 1997.

[22] J. Van der Voorde, V. Brochez, and B. Vanheel, "Heterogeneous effects of histamine on isolated rat coronary arteries," European Journal of Pharmacology, vol. 271, no. 1, pp. 17-23, 1994.

[23] C. Gonzales-Munoz, S. Nieto-Ceron, J. Cabezas-Herrera, and J. Hernandez-Cascales, "Glucagon increases contractility in ventricle but not in atrium of the rat heart," European Journal of Pharmacology, vol. 587, no. 1-3, pp. 243-247, 2008.

[24] Y. C. Kaplan, N. Hocaoglu, K. Oransay, S. Kalkan, and Y. Tuncok, "Effect of glucagon on amitriptyline-induced cardiovascular toxicity in rats," Human \& Experimental Toxicology, vol. 22, no. 4, pp. 321-325, 2008

[25] M. Rosic, I. Andjelkovic, and B. Zlokovic, "Characterization of ${ }^{3} \mathrm{H}$ histamine transport at sarcolemal membrane of the isolated perfused guinea-pig heart in the presence of glucagon and $\mathrm{H}_{1}$ and $\mathrm{H}_{2}$ receptor antagonists," Biomedical and Biochemical Acta, vol. 46, no. 10, pp. 736-740, 1987.

[26] M. Rosic, I. Andjelkovic, B. Zlokovic, et al., "Effects of glucagon and $\mathrm{H}_{1}$ and $\mathrm{H}_{2}$ receptor antagonists on ${ }^{3} \mathrm{H}$ histamine transport at sarcolemmal membrane of the isolated perfused guinea pig heart," Yugoslav Physiological and Pharmacological Acta, vol. 24, supplement, p. 391, 1988.

[27] I. Andjelkovic and B. Zlokovic, "Protective effects of glucagon during the anaphylactic response in guinea-pig isolated heart," British Journal of Pharmacology, vol. 76, no. 3, pp. 483-489, 1982.

[28] C. Prinz, R. Zanner, M. Gerhard, et al., "The mechanism of histamine secretion from gastric enterochromaffin-like cells," American Journal of Physiology, vol. 277, no. 5, pp. C845-C855, 1999.

[29] P. A. Shore, A. Burkhalter, and V. H. Cohn JR., "A method for the fluorometric assay of histamine in tissues," The Journal of Pharmacology and Experimental Therapeutics, vol. 127, pp. 182-186, 1959.

[30] L. C. Green, D. A. Wagner, J. Glogowski, P. L. Skipper, J. S. Wishnok, and S. R. Tannenbaum, "Analysis of nitrate, nitrite, and $\left[{ }^{15} \mathrm{~N}\right]$ nitrate in biological fluids," Analytical Biochemistry, vol. 126, no. 1, pp. 131-138, 1982.

[31] H. Ohkawa, N. Ohishi, and K. Yagi, "Assay for lipid peroxides in animal tissues by thiobarbituric acid reaction," Analytical Biochemistry, vol. 95, no. 2, pp. 351-358, 1979.

[32] W. Droge, "Free radicals in the physiological control of cell function," Physiological Reviews, vol. 82, no. 1, pp. 47-95, 2002.

[33] H. Farghali, J. Hodis, N. Kutinova-Canova, P. Potmesil, E. Kmonickova, and Z. Zidek, "Glucose release as a response to glucagon in rat hepatocyte culture: involvement of $\mathrm{NO}$ signaling," Physiological Research, vol. 57, no. 4, pp. 569-575, 2008.

[34] M. M. Kostic and J. Schrader, "Role of nitric oxide in reactive hyperemia of the guinea pig heart," Circulation Research, vol. 70, no. 1, pp. 208-212, 1992.

[35] R. Schulz, M. Kelm, and G. Heusch, "Nitric oxide in myocardial ischemia/reperfusion injury," Cardiovascular Research, vol. 61, no. 3, pp. 402-413, 2004.

[36] M. Kostic and V. Jakovljevic, "Role of histamine in the regulation of coronary circulation," Physiological Research, vol. 45, no. 4, pp. 297-303, 1996. 


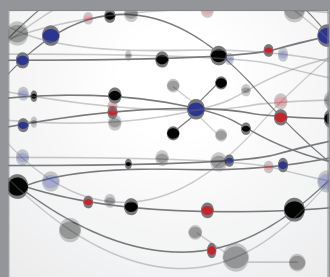

The Scientific World Journal
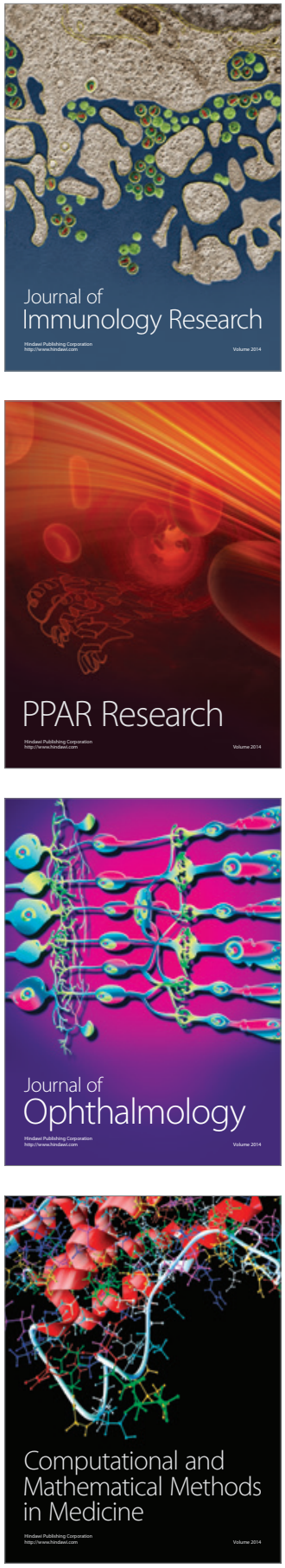

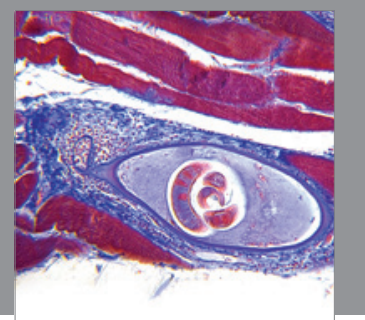

Gastroenterology

Research and Practice
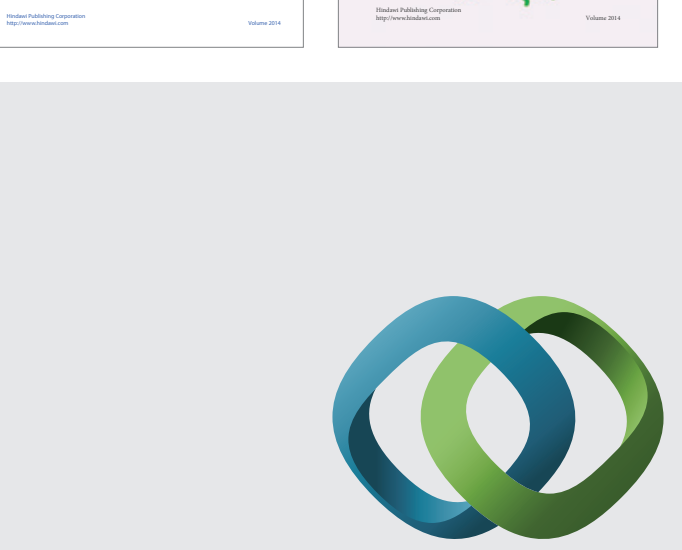

\section{Hindawi}

Submit your manuscripts at

http://www.hindawi.com
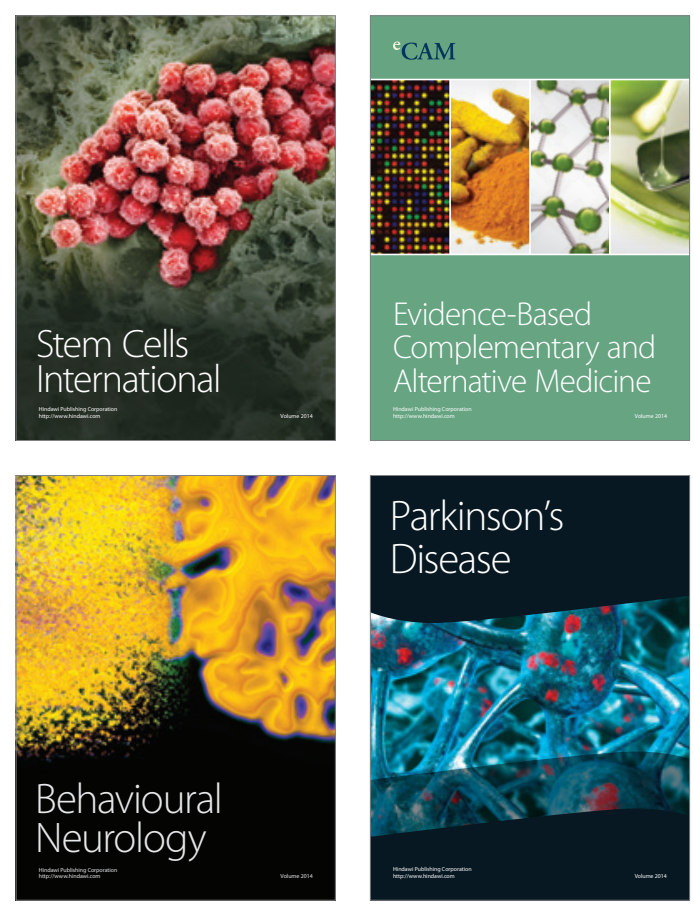

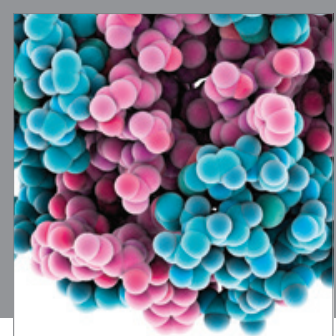

Journal of
Diabetes Research

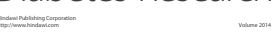

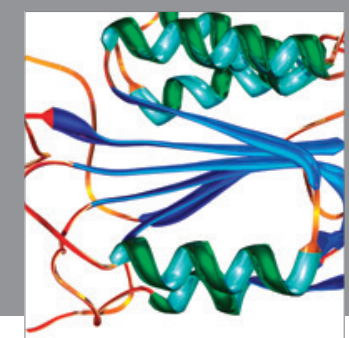

Disease Markers
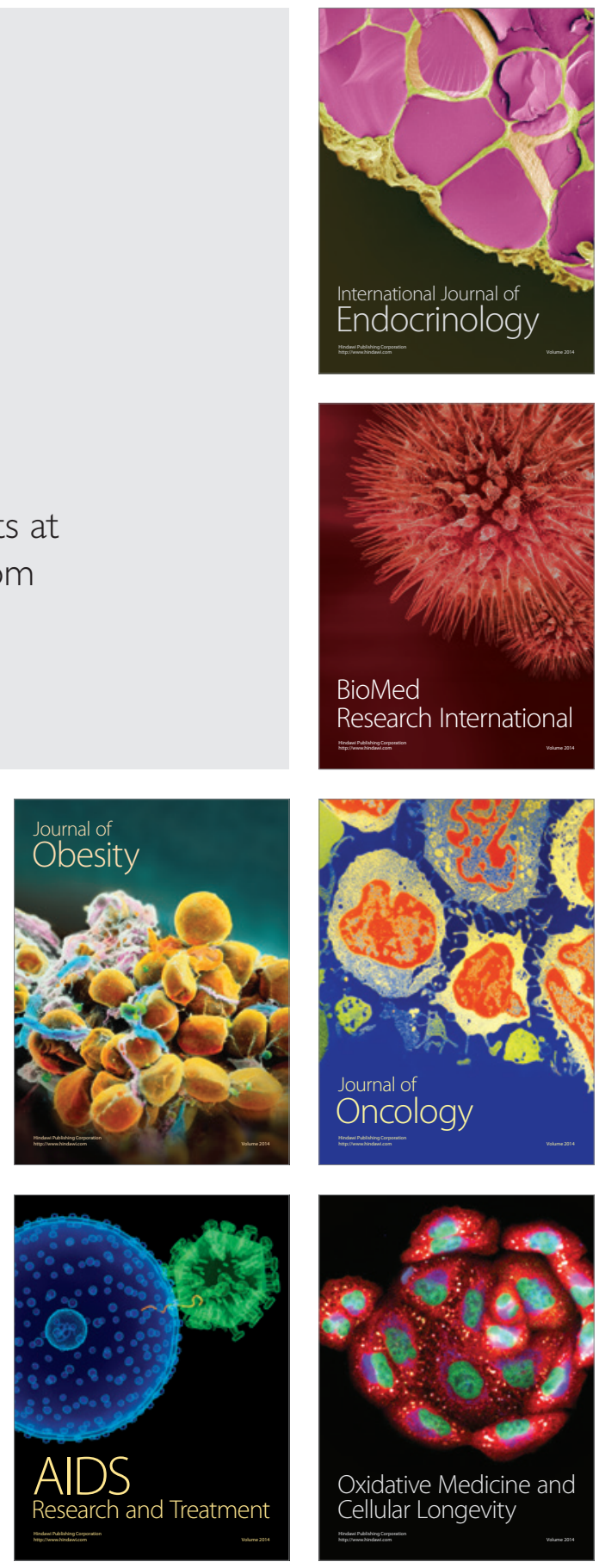